\title{
A V-INTEGRAL REPRESENTATION FOR THE CONTINUOUS LINEAR OPERATORS ON SPACES OF CONTINUOUSLY DIFFERENTIABLE VECTOR-VALUED FUNCTIONS
}

\author{
J. R. EDWARDS AND S. G. WAYMENT
}

Abstract. Suppose $X$ and $Y$ are linear normed spaces, and $C_{1}$ is the space of continuously differentiable functions from $[0,1]$ into $X$. The authors give a represention theorem for the linear operators from $C_{1}$ into $Y$ in terms of the $v$-integral operating on the function as opposed to the derivative of the function.

1. Introduction. Let $C_{1}$ denote the space of continuously differentiable functions on the interval $[0,1]$ with values in a linear normed space $X$, and let $C_{1}$ be normed by $\|f\|_{1}=\|f\|_{\infty}+\left\|f^{\prime}\right\|_{\infty}$. In the special case that $X$ is the real numbers $R$, we denote the function space by $C_{1} R$. The following characterization of the continuous linear functionals $T$ on $C_{1} R$ is given in $[1$, Chapter 14, B. $36(\mathrm{a})$, p. 344]: $T(f)$ $=\alpha f(0)+\int_{0}^{1} f^{\prime} d \mu$, where $\alpha$ is scalar and $\mu$ is a regular borel measure. Employing the results in [6], one obtains the following representation (but not a characterization unless $Y$ is weakly complete) of the continuous linear operators from $C_{1}$ into a linear normed space $Y: T(f)$ $=\Psi(f(0))+\int d K \cdot f^{\prime}$, where $\Psi$ is an element of $B[X, Y]$, the continuous linear operators from $X$ in to $Y$, and $K$ is a finitely additive operator valued set function from the half open intervals into $B\left[X, Y^{+}\right]$which is weakly regular and of bounded semivariation $\left(Y^{+}\right.$ denotes the weak sequential extension of $Y$ as defined in $[6, \S 2])$.

Another representation (which gives a characterization under the less restrictive condition that $Y$ is complete) of these linear operators follows from [5]: $T(f)=\Psi_{1}(f(0))+\Psi_{2}\left(f^{\prime}(0)\right)+v \int K \cdot d f^{\prime}$, where $\Psi_{1}$ and $\Psi_{2}$ are elements of $B[X, Y]$ and the $v$-integral is the generalized Stieltjes-type integral of a convex operator valued set function which is convex-Gowurin and defined from the half open intervals into $B[X, Y]$, as defined in [4]. Since each of the three mentioned representations use the fact that $C_{1}$ can be decomposed into $X \oplus C$ (using Lemma 2.3 of this paper), each shares the shortcoming that the integrals operate on $f^{\prime}$ rather than on $f$. In this paper we use the $v$ -

Received by the editors April 9, 1970.

AMS 1969 subject classifications. Primary 2825, 4725; Secondary 4630.

Key words and phrases. Generalized Stieltjes-type integral, convex with respect to length, 1-Gowurin, differentiable vector-valued functions, weak sequential extension. 
integral to give a representation which involves $f$ directly, rather than requiring one to compute $f^{\prime}$.

For convenience we let $C_{1,0}$ denote the subspace of functions $f$ from $C_{1}$ such that $f(0)=0_{X}$, the additive identity in $X$. Let $P$ denote the polygonal functions which are $0_{X}$ at 0 , and observe that $\|\cdot\|_{1}$ can be extended to $C_{1,0}+P$ by

$$
\|f+p\|_{1}=\|f+p\|_{\infty}+\left\|f^{\prime}+p^{\prime}\right\|_{\text {ess, }}
$$

where $f \in C_{1,0}, p \in P$, and $\|\cdot\|_{\text {ess }}$ denotes the essential supremum norm. For each $f \in C_{1,0}$ and partition $\sigma$ of $[0,1]$, we define the polygonal function $p f_{\sigma}$ to have corners precisely at the points $t_{i}$ where $\sigma=\left\{t_{i}\right\}_{i=1}^{n}$ and such that $p f_{\sigma}\left(t_{i}\right)=f\left(t_{i}\right)$ for each $i$.

Let $g$ denote the collection of half open intervals $(a, b]$. A set function $K$ from $g$ into $B[X, Y]$ is said to be convex with respect to length provided that $K(E)=\sum_{i=1}^{n} \lambda_{i} K\left(E_{i}\right)$ whenever $\bigcup_{i=1}^{n} E_{i}=E$ and $E_{i}$ $\cap E_{j}=\varnothing$ for $i \neq j$, and where $\lambda_{i}=l\left(E_{i}\right) / l(E)$, that is, the ratio of the length of $E_{i}$ to the length of $E$. Such a function is said to be 1Gowurin if there exists a constant $W K$ such that for each finite collection of disjoint intervals $\left\{E_{i}\right\}_{i=1}^{n}$ and collection $\left\{x_{i}\right\}_{i=1}^{n}$ of elements from $X$,

$$
\left\|\sum\left[K\left(E_{i}\right)\right]\left(x_{i}\right)\right\|_{Y} \leqq W K\left\{\max _{j}\left\|\sum_{i=1}^{j} x_{i}\right\|+\max _{j}\left\|x_{j}\right\| / l\left(E_{j}\right)\right\} .
$$

The general procedure for obtaining the representation theorem for $T$ is as follows. First it is established that $\lim _{|\sigma|} p f_{\sigma}=f$ for each $f \in C_{\mathbf{1}, 0}$. Then the polygonal functions are decomposed into linear sums of fundamental functions. The fundamental functions are not in $C_{1,0}$ but are imbedded along with $C_{1,0}$ into $C_{1,0}^{+}$, the weak sequential extension of $C_{\mathbf{1}, 0}$. This is accomplished with a continuous linear operator $\theta$ from $C_{1,0}+P$ into $C_{1,0}^{+}$. Then, the linear transformation $T$ is extended to $T^{+}$ on $C_{1,0}^{+}$in the natural way. Finally $T^{+}$is composed with the $\theta$ map and the action on the fundamental functions determines the convex set function $K$ necessary to represent $T$ in terms of $v$-integration of $K$ with respect to $f$. Thus the procedure is entirely analogous to that described in [3] to obtain representation theorems in terms of more classical-type integration processes.

2. Preliminary results. The principal result in this section is the following theorem.

2.1. Theorem. Suppose $f$ is an element of $C_{1}$. Then $\left\{p f_{\sigma}\right\}$ converges to $f$ in the 1-norm as $|\sigma|$ converges to zero. 
Before proving Theorem 2.1, we first establish the following results for the calculus of vector functions. The first lemma is fundamental to Theorem 2.1, and in its proof we use the sliding interval technique [2].

2.2. Lemma. If $f$ is a differentiable $X$-valued function, and $f^{\prime}(t)=\theta_{X}$ for all $t$, then $f$ is a constant.

Proof. Under the above hypothesis assume that $f$ is not constant. Then, there are numbers $a, b \in[0,1]$ such that $(f(b)-f(a)) /(b-a)$ $\neq 0_{X}$. Define $c=(b+a) / 2$, then one of $\|(f(b)-f(c)) /(b-c)\|$ and $\|(f(c)-f(a)) /(c-a)\|$ must have norm at least as large as $\alpha$ $=\|(f(b)-f(a)) /(b-a)\|$. Let $\left[a_{1}, b_{1}\right]$ denote the interval on which this holds. Proceed inductively constructing a nested sequence of intervals $\left\{\left[a_{n}, b_{n}\right]\right\}$ such that, for each $n,\left(b_{n}-a_{n}\right)=\frac{1}{2}\left(b_{n+1}-a_{n+1}\right)$ and $\left\|\left(f\left(b_{n+1}\right)-f\left(a_{n+1}\right)\right) /\left(b_{n+1}-a_{n+1}\right)\right\| \geqq \alpha$. Let $x_{0}=\bigcap_{n=1}^{\infty}\left[a_{n}, b_{n}\right]$. Then it follows that $f^{\prime}\left(x_{0}\right) \neq 0_{X}$ contrary to hypothesis. Therefore, we conclude that $f$ must be constant, and the lemma is established.

2.3. Lемма. If $f$ is a continuously differentiable $X$-valued function, then $f(t)=f(0)+\int_{0}^{t} f^{\prime}(s) d s$.

Proof. It follows as in the classical setting that $g^{\prime}(t)=f^{\prime}(t)$ where $g(x)=\int_{0}^{x} f^{\prime}(t) d t$. Therefore, $f^{\prime}(t)-g^{\prime}(t)=0_{X}$ for each $t$ from which it follows that $f(t)-g(t)$ is identically a constant, which is given by $f(0)-g(0)=f(0)$.

2.4. LeмmA. If $f$ is a continuously differentiable $X$-valued function, then the difference functions

$$
f_{h_{1} h_{2}}(t)=\left(f\left(t+h_{1}\right)-f\left(t-h_{2}\right)\right) /\left(h_{1}+h_{2}\right), \quad h_{1}, h_{2}>0,
$$

converge uniformly to $f^{\prime}$ as $h_{1}+h_{2} \rightarrow 0$.

Proof. $f^{\prime}$ is uniformly continuous on $[0,1]$; hence, given $\epsilon>0$ there is a $\delta>0$ such that if $\left|t-t^{\prime}\right|<\delta$, then $\left\|f^{\prime}(t)-f^{\prime}\left(t^{\prime}\right)\right\|<\epsilon$. Choose $h_{1}+h_{2}$ $<\delta$. Then,

$$
\begin{aligned}
\left\|f_{h_{1} h_{2}}(t)-f^{\prime}(t)\right\| & =\left\|\left(h_{1}+h_{2}\right)^{-1} \int_{t-h_{2}}^{t+h_{1}}\left(f^{\prime}(s)-f^{\prime}(t)\right) d s\right\| \\
& \leqq\left(h_{1}+h_{2}\right)^{-1}\left(\epsilon\left(h_{1}+h_{2}\right)\right)=\epsilon,
\end{aligned}
$$

from which the lemma follows.

Proof of 2.1. Suppose $\epsilon>0$. Lemma 2.4 implies that there is a $\delta>0$ such that if $h_{1}+h_{2}<\delta, h_{1}, h_{2}>0$, then $\left\|f_{h_{1}, h_{2}}-f^{\prime}\right\|_{\infty}<\epsilon / 2$. Suppose $\sigma$ is a partition whose mesh-fineness, $|\sigma|$, is less than $\delta$. Let $\left[t_{i}, t_{i+1}\right]$ denote one of the intervals defined by $\sigma$. Then $\left(p f_{\sigma}\right)^{\prime}$ on $\left(t_{i}, t_{i+1}\right)$ is 


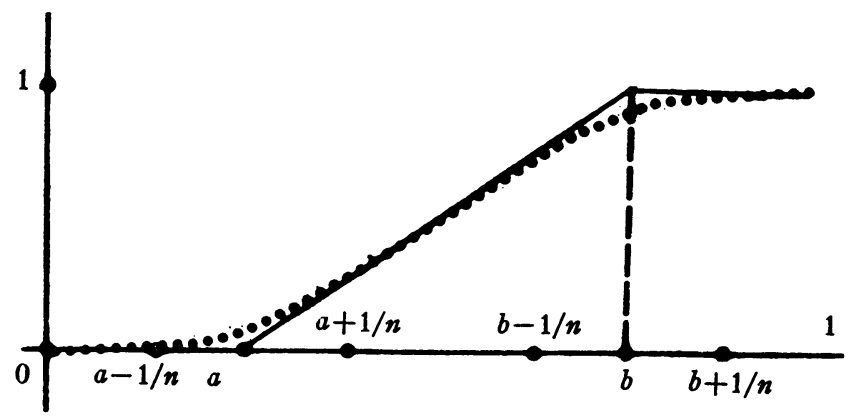

Figure 1a
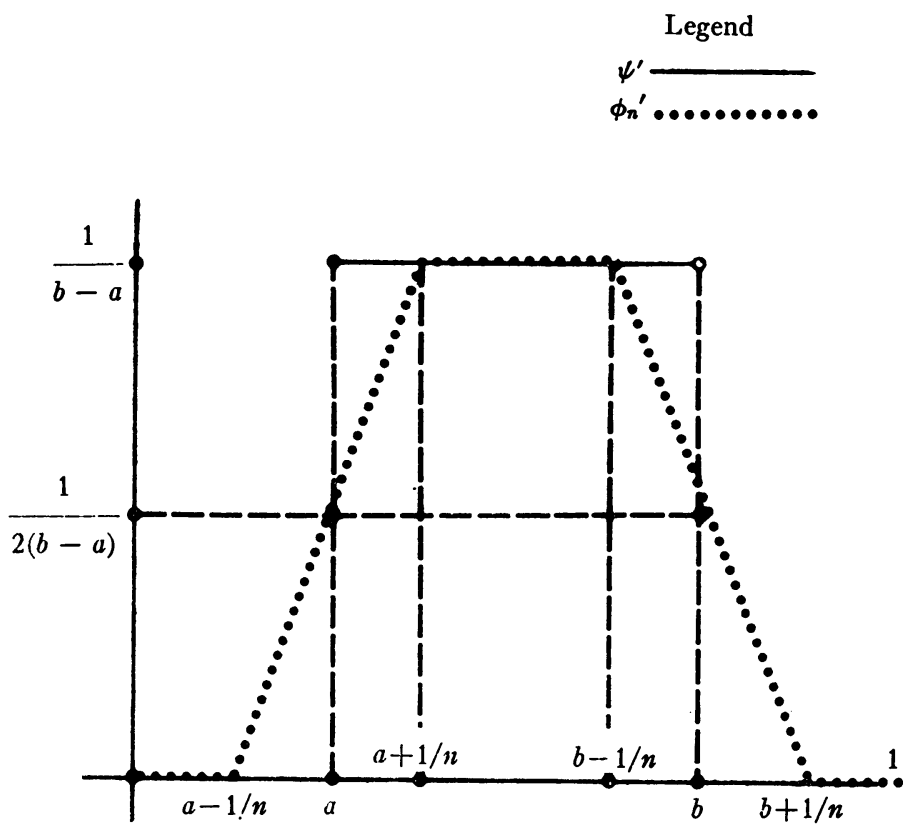

Figure 1b

given by $\left(f\left(t_{i+1}\right)-f\left(t_{i}\right)\right) /\left(t_{i+1}-t_{i}\right)$. Let $t \in\left(t_{i}, t_{i+1}\right)$ and define $h_{1}=t_{i+1}$ $-t$ and $h_{2}=t-t_{i}$. Then $\left(p f_{\sigma}\right)^{\prime}(t)=f_{h_{1}, h_{2}}(t)$ and it follows that $\left\|\left(p f_{\sigma}\right)^{\prime}(t)-f^{\prime}(t)\right\|<\epsilon / 2 \quad$ since $\quad h_{1}+h_{2}=t_{i+1}-t_{i}<\delta$. Therefore, 
$\left\|\left(p f_{\sigma}\right)^{\prime}-f^{\prime}\right\|_{\text {ess }}<\epsilon / 2$, from which we conclude $\left\|p f_{\sigma}-f\right\|_{\infty}<\epsilon / 2$ and, hence, that $\left\|p f_{\sigma}-f\right\|_{1}<\epsilon$. The theorem is proved.

3. The $\theta$-map. In this section we construct a canonical, continuous linear mapping from $C_{1,0}+P$ into $C_{1,0}^{+}$. Suppose $(a, b] \in g$. As in [4], let $\psi_{(a, b]}$ denote the fundamental function of the interval $(a, b]$ defined by $\psi_{(a, b]}(t)=0$ if $0 \leqq t \leqq a, \psi_{(a, b]}(t)=(t-a) /(b-a)$ if $a \leqq t \leqq b$, and $\psi_{(a, b]}(t)=1$ if $b \leqq t \leqq 1$. Define the sequence of continuous functions $\left\{\Phi_{n}\right\}$ by $\Phi_{n} \equiv 0$ if one of the following hold: $1 / n \geqq a, 1 / n \geqq 1-b$, or $2 / n \geqq b-a$; and otherwise by

$$
\begin{array}{ll}
\Phi_{n}(x)=0 & \text { for } 0 \leqq x \leqq a-1 / n, \\
\Phi_{n}(x)=\left[n / 2(x-a+1 / n)^{2}\right] / 2(b-a) & \text { for } a-1 / n \leqq x \leqq a+1 / n, \\
\Phi_{n}(x)=\psi_{(a, b]}(x) & \text { for } a+1 / n \leqq x \leqq b-1 / n, \\
\Phi_{n}(x)=1-[1-(n / 2)(t-b+1 / n)]^{2} / n(b-a) & \text { for } b-1 / n \leqq x \leqq b+1 / n,
\end{array}
$$

and

$$
\Phi_{n}(x)=1 \text { for } b+1 / n \leqq x \leqq 1 .
$$

(See Figures 1a and 1b.)

Then $\left\{\Phi_{n}\right\}$ is a sequence in $C_{1} R$ which is uniformly bounded and satisfies the properties that $\left\{\Phi_{n}\right\}$ converges pointwise to $\psi_{(a, b]}$, that $\left\{\Phi_{n}{ }^{\prime}\right\}$ converges pointwise to $\Psi_{(a, b]}$ which is defined by $\Psi_{(a, b]}(x)=\psi^{\prime}(x)$ for $x \neq a$ or $b$ and $\psi(x)=1 / 2(b-a)$ for $x=a$ or $b$. It follows from 13.36 $\left[1\right.$, p. 345] that $\left\{\Phi_{n}\right\}$ is weakly convergent in $C_{1} R$. Hence, for $x \in X$ it follows that $\left\{\Phi_{n} \cdot x\right\}$ is weakly convergent in $C_{1,0}$. Let $\psi[(a, b], x]$ denote the weak limit of $\left\{\Phi_{n} \cdot x\right\}$ in $C_{1}$. Define $\theta$ from $C_{1}+P$ by $\theta\left(f+\sum_{i} \psi_{E_{i}} \cdot x_{i}\right)=f+\sum \psi\left[E_{i}, x_{i}\right]$ where $f \in C_{1}$ and $\sum_{i} \psi_{E_{i}} \cdot x_{i} \in P$.

\subsection{Lemma. The linear map $\theta$ is continuous.}

In order to show $\theta$ is continuous it is sufficient to show that if $\left\{p_{n}\right\}$ is a sequence of polygonal functions converging to a function $f$ in $C_{1,0}$ in the 1 -norm, that $\left\{\theta\left(p_{n}\right)\right\}$ converges to $f$ in $C_{1,0}^{+}$. A convenient tool to demonstrate such convergence is the following lemma which is established in [3].

3.2. Lemma. Suppose $S$ is a linear normed space. A sufficient condition that a sequence $\left\{s_{n}^{+}\right\}$in $S^{+}$converges to $s_{0}^{+} \in S^{+}$is that, for each $n=0,1, \cdots$, there exists a sequence $\left\{s_{n, m}\right\}_{m=1}^{\infty}$ in $S$ such that

(a) for each $n,\left\{s_{n, m}\right\}$ converges weakly to $s_{n}^{+}$;

(b) $\left\|s_{n, m}-s_{0, m}\right\|$ converges to 0 as $n, m$ increase without bound. 
Proof of 3.1. Suppose $\left\{p_{n}\right\} \subset P$ converges to $f \in C$ in the 1 -norm. For each $n, p_{n}$ has the form $\sum_{i=1}^{\nu(n)} \psi_{E_{i}}^{n} \cdot x_{i}^{n}$. Suppose $\epsilon$ is a positive number. For each pair $(n, i)$ let $\left\{\Phi_{i, m}^{n}\right\}$ denote the sequence defined relative to $\psi_{E_{i}}^{n}$ as in this section. Then, $\left\{\sum_{i=1}^{\nu(n)} \Phi_{i, m}^{n} \cdot x_{i}^{n}\right\}$ converges weakly to $\theta\left(p_{n}\right)$ for each $n$. For each $n$ define the sequence $\left\{h_{n, m}\right\}_{m=1}^{\infty}$ by $h_{n, m}=\sum_{i=1}^{\nu(n)} \Phi_{i, n(m)}^{n} \cdot x_{i}^{n}$ where $\{n(m)\}$ is a sequence of integers (depending on $n$ ) such that $\Phi_{i, n(1)}^{n}$ is not identically zero for $i=1$, $2, \cdots, \nu(n)$ and such that $n(\nu+1)=n(\nu)+1$. It follows that for each $n\left\{h_{n, m}\right\}_{m=1}^{\infty}$ converges weakly to $\theta\left(p_{n}\right)$. Since $\left\{p_{n}\right\}$ converges to $f$ in the 1 -norm, there is an $N$ such that $n \geqq N$ and $\left\|p_{n}{ }^{\prime}-f^{\prime}\right\|_{\text {ess }}<\epsilon / 5$. Since $f^{\prime}$ is uniformly continuous, then there is a $\delta$ such that if $\left|x-x^{\prime}\right|<\delta$, then $\left\|f^{\prime}(x)-f^{\prime}\left(x^{\prime}\right)\right\|<\epsilon / 5$. Choose $M$ such that $2 / M<\delta$. Choose $n>N$ and $m>M$. Let $\left\{t_{i, n}\right\}_{i=1}^{\nu(n)}$ denote the domain values of the corner points of $p_{n}$. Then,

$$
\left\|f^{\prime}(t)-h_{n, m}^{\prime}(t)\right\|=\left\|f^{\prime}(t)-p_{n}^{\prime}(t)\right\|<\epsilon / 5,
$$

for $t$ in the complement of $\bigcup_{i=1}^{\nu(n)}\left[t_{i, n}-1 / n(m), t_{i, n}+1 / n(m)\right]$. Suppose $t \in\left(t_{j, n}-1 / n(m), t_{j, n}+1 / n(m)\right)$. Then,

$$
\begin{aligned}
\left\|f^{\prime}(t)-h_{n, m}^{\prime}(t)\right\| \leqq & \left\|f^{\prime}(t)-f^{\prime}\left(t_{j, n}-1 / n(m)\right)\right\| \\
& +\left\|f^{\prime}\left(t_{j, n}-1 / m(n)\right)-p_{n}^{\prime}\left(t_{j, n}-1 / n(m)\right)\right\| \\
& +\left\|p_{n}^{\prime}\left(t_{j, n}-1 / n(m)\right)-h_{n, m}^{\prime}(t)\right\| .
\end{aligned}
$$

However,

$$
\begin{aligned}
&\left\|p_{n}^{\prime}\left(t_{j, n}-1 / n(m)\right)-h_{n, m}^{\prime}(t)\right\| \\
& \leqq\left\|p_{n}^{\prime}\left(t_{j, n}-1 / n(m)\right)-p_{n}^{\prime}\left(t_{j, n}+1 / n(m)\right)\right\| \\
& \leqq\left\|p_{n}^{\prime}\left(t_{j, n}-1 / n(m)\right)-f^{\prime}\left(t_{j, n}-1 / n(m)\right)\right\| \\
&+\left\|f^{\prime}\left(t_{j, n}-1 / n(m)\right)-f^{\prime}\left(t_{j, n}-1 / n(m)\right)\right\| \\
&+\left\|f^{\prime}\left(t_{j, n}+1 / n(m)\right)-p^{\prime}\left(t_{j, n}+1 / n(m)\right)\right\| \\
&< \epsilon / 5+\epsilon / 5+\epsilon / 5 .
\end{aligned}
$$

It follows that $\left\|f^{\prime}(t)-h_{n, m}^{\prime}(t)\right\|<\epsilon / 5+\epsilon / 5+3(\epsilon / 5)=\epsilon$, and 2.3 implies that $\left\|f-h_{n, m}\right\|<\epsilon$. Therefore, 3.2 is satisfied, and it follows that $\left\{\theta\left(p_{n}\right)\right\}$ converges to $f$. Hence $\theta$ is continuous.

\subsection{LemMa. The norm of the restriction of $\theta$ to $P$ is 1 .}

Proof. It is sufficient to show that $\theta$ is norm-nonincreasing. Suppose $p=\sum \psi_{E_{i}} x_{i} \in P$. Then, $\left\{\sum \Phi_{i, m} x_{i}\right\}$ converges weakly to $\theta\left(p_{n}\right)$, 
where for each $i,\left\{\Phi_{i, m}\right\}$ is the sequence constructed in this section. Then

$$
\begin{aligned}
\|\theta(p)\|_{+} & =\sup \left\{\lim \left|\left\langle s^{*}, \sum \Phi_{i, m} x_{i}\right\rangle\right|: s^{*} \in C_{1}^{*} \text { and }\left\|s^{*}\right\|=1\right\} \\
& \leqq \sup \left\{\limsup \left\|s^{*}\right\|\left\|\sum \Phi_{i, m} x_{i}\right\|_{1}:\left\|s^{*}\right\|=1\right\} \\
& <\lim \sup _{m}\left\|\sum_{i} \Phi_{i, m} x_{i}\right\|_{\infty}+\underset{m}{\lim \sup }\left\|\sum_{i} \Phi_{i, m}^{\prime} x_{i}\right\|_{\infty} \\
& =\left\|\sum_{i} \psi_{E_{i}} x_{i}\right\|_{\infty}+\left\|\sum_{i} \psi_{E_{i}}^{\prime} x_{i}\right\|_{\text {ess }}=\left\|\sum_{i} \psi_{E_{i}} x_{i}\right\|_{1} .
\end{aligned}
$$

\section{The representation theorem.}

4.1. Theorem. Suppose $T$ is a continuous linear operator from $C_{1}$ into $Y$. Then there is a linear operator $\Phi$ from $X$ into $Y$ and a set function $K$ from $g$ into $B\left[X, Y^{+}\right]$which is convex with respect to length and which is 1-Gowurin such that $T(f)=\Phi(f(0))+v \int K d f$. Furthermore, the norm of $T$ restricted to $C_{1,0}$ is equal to $W K$, where $W K$ is the smallest constant satisfying inequality (1) from the Introduction of this paper.

Proof. We recall that the historical technique for obtaining an integral representation theorem for an operator $T$ has been to extend the function space to include (usually in a weak sequential extension sense) the step functions or simple functions, which are dense in the extended norm on the extended function space. The action of the operator $T$ on the step or simple functions determines an additive set function and finally approximation of a function by a linear combination of step or simple functions leads to an integral representation of the operator. Since we have continuously imbedded $C_{1,0}+P$ into $C_{1,0}^{+}$ with the $\theta$ map, we are now in a position to follow an analogous procedure where fundamental functions replace step or simple functions and the $C_{1}$ norm replaces sup norm. Consider $T$ restricted to $C_{1,0}$. Then $T$ has a natural extension from $C_{1,0}^{+}$in to $Y^{+}$which we denote by $T^{+}$. Define the set function $K$ from $g$ into $B\left[X, Y^{+}\right]$by $[K(E)](x)$ $=T^{+}\left(\theta\left(\psi_{E} X\right)\right)$. Then for $f \in C_{1,0}$ we have

$$
\begin{aligned}
T(f) & =T^{+}(\theta(f))=T^{+}\left(\theta\left(\lim _{|\sigma| \rightarrow 0} p f_{\sigma}\right)\right)=\lim _{|\sigma| \rightarrow 0} T^{+}\left(\theta\left(p f_{\sigma}\right)\right) \\
& =\lim _{|\sigma| \rightarrow 0} T^{+}\left(\theta\left(\sum \Delta_{i} f \psi_{E_{i}}\right)\right)=\lim _{|\sigma| \rightarrow 0} \sum K\left(E_{i}\right) \Delta_{i} f=v \int K d f .
\end{aligned}
$$


If the interval $E$ is the union of the pairwise disjoint intervals $\left\{E_{i}\right\}_{i=1}^{n}$, then $\psi_{E}=\sum_{i=1}^{n}\left[l\left(E_{i}\right) / l(E)\right] \psi_{E_{i}}$ and consequently $K(E)$ $=\sum_{i=1}^{n} \lambda_{i} K\left(E_{i}\right)$ and $K$ is convex with respect to length. It is straightforward and follows as in Theorem 4.2 in [4] that $K$ is 1 -Gowurin and that $\left\|T^{+} \theta\right\| \geqq W K$. However, since $\theta$ is the identity map on $C_{1,0}$ and since the norm of $\theta$ on $P$ is 1 , we conclude that $\|T\|=W K$.

In general the converse of 4.1 does not hold unless $Y$ is weakly sequentially complete. However, a partial converse does hold, and since its proof follows essentially along the lines of the proof of Theorem 4.2 in [4] we state the result without proof.

4.2. Theorem. Suppose $K$ is a set function from $g$ with values in $B[X, Y]$. Then, $T(f)=v \int K d f$ is a continuous linear operator from $C_{1,0}$ into $Y$. Furthermore, $\|T\|=W K$.

4.3. Example. Suppose $x_{0} \in(0,1)$. The convex set function $K: g$ $\rightarrow \boldsymbol{R}$ defined by $K(a, b]=0$ if $x_{0} \in(0, a) \cup(b, 1), K(a, b]=1 /(b-a)$ if $x_{0} \in(a, b)$, and $K(a, b]=1 / 2(b-a)$ if $x=a$ or if $x=b$, yields the transformation $T(f)=v \int K d f=f^{\prime}\left(x_{0}\right)$.

4.4. ExAmple. The convex set function $K: g \rightarrow B\left[R, C^{+}\right]=C^{+}$ given by $[K(a, b]](x)=0$ if $x \in(0, a) \cup(b, 1),[K(a, b]](x)=1 /(b-a)$ if $x \in(a, b)$, and $[K(a, b]](x)=1 / 2(b-a)$ if $x=a$ or if $x=b$ yields the transformation $T(f)=\int K d f=f^{\prime}$.

4.5. Remark. Suppose $f$ is differentiable $n$ times. Then to obtain $f^{(n)}(x)$ one applies $4.4(n-1)$ times and then 4.3.

\section{BIBLIOGRAPHY}

1. N. Dunford and J. T. Schwartz, Linear operators. I: General theory, Pure and Appl. Math., vol. 7, Interscience, New York, 1958. MR 22 \#8302.

2. R. J. Easton and S. G. Wayment, The sliding interval technique, Amer. Math. Monthly 75 (1968), 886-888.

3. J. R. Edwards and S. G. Wayment, A unifying representation theorem, Math. Ann. 187 (1970), 317-328.

4. - Representations for transformations continuous in the BV norm, Trans. Amer. Math. Soc. 154 (1971), 251-265.

5. - A v-integral representation for linear operators on a space of continuous vector-valued functions, Proc. Amer. Math. Soc. 30 (1971), 260-262.

6. D. H. Tucker, $A$ representation theorem for a continuous linear transformation on a space of continuous functions, Proc. Amer. Math. Soc. 16 (1965), 946-953. MR 33 \#7865.

Utar State University, Logan, Utah 84321 